\title{
Correction to: The Existence of Solutions for a Nonlinear First-Order Differential Equation Involving the Riemann-Liouville Fractional-Order and Nonlocal Condition
}

Fatma M. Gaafar

Correction to: Mediterr. J. Math. (2018) 15:191 https://doi.org/10.1007/s00009-018-1236-3

In the original publication, the affiliation of the author was incorrectly written. The correct affiliation is: Department of Mathematics, Faculty of Science, Damanhour University, Beside Damanhour General Hospital, El Gomhoureya St., Damanhour, Egypt.

I regret for the mistake.

Publisher's Note Springer Nature remains neutral with regard to jurisdictional claims in published maps and institutional affiliations.

Fatma M. Gaafar

Department of Mathematics

Faculty of Science

Damanhour University

Beside Damanhour General Hospital

El Gomhoureya St.

Damanhour

Egypt

e-mail: fatmagaafar2@yahoo.com

The original article can be found online at: https://doi.org/10.1007/s00009-018-1236-3. 